\title{
GO Shaping of Omnidirectional Dual-Reflector Antennas for Maximum Gain
}

\author{
Fernando J. S. Moreira*, Aluizio Prata, Jr. ${ }^{\dagger}$, and José R. Bergmann ${ }^{\ddagger}$ \\ ${ }^{*}$ Dept. Electronics Engineering, Federal University of Minas Gerais, CEP 31270-901, MG, Brazil. \\ ${ }^{\dagger}$ Dept. Electrical Engineering-Electrophysics, University of Southern California, Los Angeles, CA 90089-0271, USA. \\ ${ }^{\ddagger}$ Center for Telecommunication Studies, Catholic University of Rio de Janeiro, CEP 22453-130, RJ, Brazil.
}

\begin{abstract}
This work presents the formulation for the reflector shaping of dual-reflector antennas designed to offer an omnidirectional coverage. The shaping is based on geometrical optics (GO) principles and assumes a uniform phase distribution for the aperture field. Two distinct dual-reflector arrangements (based on the ADC and ADE configurations) are investigated. The GO shaping results are validated by accurate analyses provided by the moment-method technique.

Index Terms-Reflector antennas, omnidirectional antennas, reflector shaping.
\end{abstract}

\section{INTRODUCTION}

Reflector antennas are widely used in microwave and millimeter-wave wireless communication systems demanding large transmission rates. That is basically due to their inherent broadband radiation characteristics, which are ultimately related to the quasi-optical behavior of the reflector arrangement. Usually, they are designed for large directivities (as those in a point-to-point microwave link), but some attention has been devoted recently to reflector arrangements providing omnidirectional coverage [1]-[9]. Omnidirectional reflector configurations are suited for operating as basestation antennas, covering the surrounding area in a broadband point-multi-point radio link.

The studies conducted cover from classical reflector arrangements (i.e., arrangements whose reflectors are generated by conic sections) [2],[7]-[9] to shaped reflectors [1],[3]-[6]. But no study has been devoted yet to the synthesis of highly-efficient omnidirectional dual-reflector antennas, which are achieved by means of a uniform aperture illumination. So, the objective of this work is to present a dual-reflector shaping procedure based on geometrical optics (GO) principles, developed to attain an aperture illumination with a uniform phase and an arbitrary amplitude distribution from a prescribed circularly symmetric feed radiation (and some pertinent geometrical parameters). The arbitrariness of the aperture field amplitude allows the application of the present GO shaping procedure in designs aiming maximum antenna gains (by choosing a uniform amplitude distribution at the aperture) or low sidelobe levels (by means of a tapered amplitude distribution).

\section{BASIC Geometrical FeAtures}

There are four different types of dual-reflector antennas capable of providing omnidirectional coverages [8], but those based on the classical axis-displaced Cassegrain (ADC) and axis-displaced ellipse (ADE) seem to be the most appropriate ones for yielding compact arrangements. Such arrangements are depicted in Figs. 1 and 2, respectively, together with some pertinent geometrical parameters of the body-of-revolution reflector surfaces. One can inspect from these figures that the main difference between the ADC- and ADE-like configurations is that the principal ray (leaving the antenna principal focus along the symmetry axis and striking the subreflector at its vertex) meets the main reflector at its inner (outer) rim in the ADC (ADE) arrangement. The essential consequence is that the ADElike reflector arrangement provides the inverse of the feed illumination toward the reflector-antenna aperture, which is an interesting feature to be taken into account by the antenna designer.

Among the antennas geometrical parameters, its useful to point out from Figs. 1 and 2 the width $\left(W_{A}\right)$ of the antenna conical aperture, the main-reflector outer $\left(D_{M}\right)$ and inner $\left(D_{B}\right)$ diameters, the subreflector diameter $\left(D_{S}\right)$, the distance $\left(V_{S}\right)$ from the antenna principal focus $O$ (which is also the origin of the coordinate system) to the subreflector vertex, the $z$-coordinate $\left(z_{B}\right)$ of the main-reflector central hole (i.e., the $z$-coordinate of point $P_{2}$ ), the subreflector edge angle $\left(\theta_{E}\right)$, and the angle $(\gamma)$ between the rays reflected by the main reflector and the symmetry axis. The angle $\gamma$ basically controls the direction of the maximum directivity of the omnidirectional antenna radiation pattern. For instance, if the usual directive configurations are desired, one just need to set $\gamma=0$. Another important parameter is the equal path length $\left(\ell_{o}\right)$ from the principal focus $O$ to the aperture point $A$, which comes as a consequence of the desired uniform phase distribution over the antenna aperture.

\section{GO SHAPING FORMULATION}

Many GO reflector-shaping algorithms have been developed since Galindo's pioneer work [10]. But for the present task we derive one based on a shaping procedure originally developed for directive circularly-symmetric dual-reflector antennas [11]. The procedure is similar to that presented in [12] but applying Fermat's principle instead of Snell's law to treat the reflection at the subreflector's surface.

Always with the help of Figs. 1 and 2, let $\overline{O S M}$ be a certain optical path length departing from $O$, reflecting 


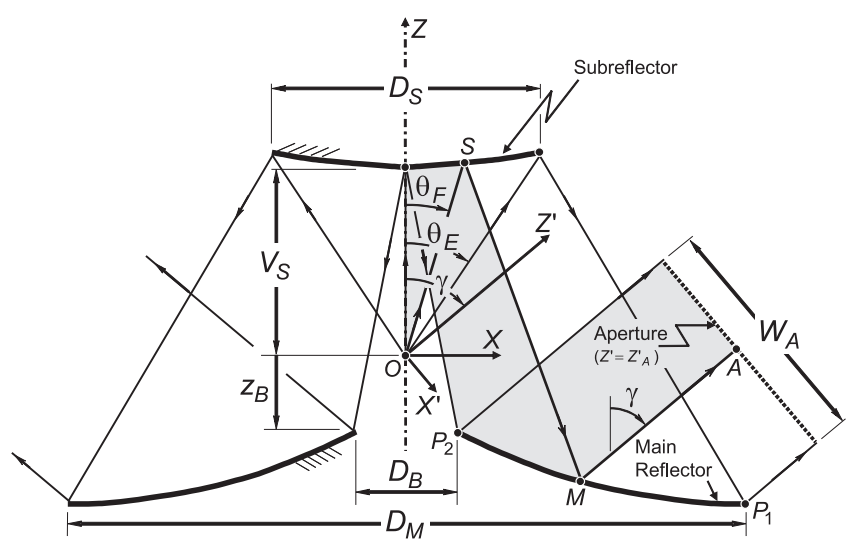

Fig. 1. Geometry of the ADC-like omnidirectional dual-reflector antenna.

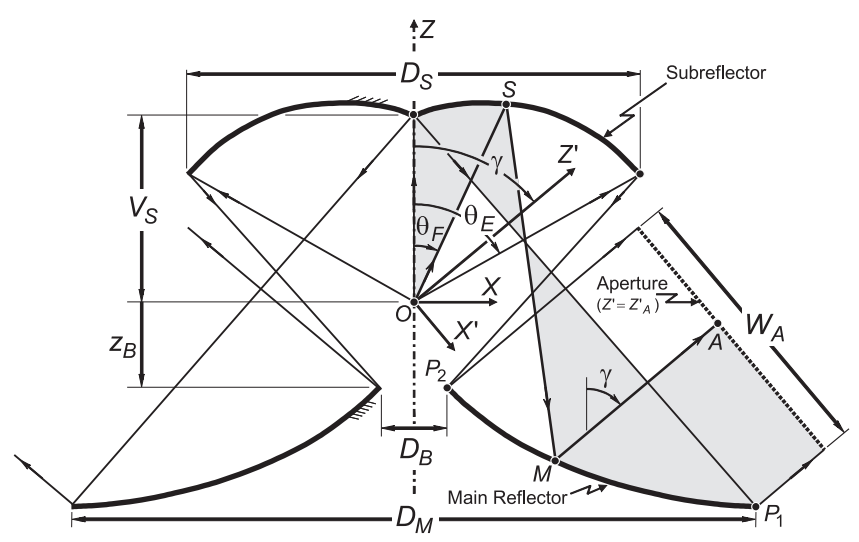

Fig. 2. Geometry of the ADE-like omnidirectional dual-reflector antenna.

at the subreflector surface at $S$ and arriving at the mainreflector point $M$, before continuing toward the antenna aperture. Such path must then obey Fermat's principle (and, consequently, Snell's law at $S$ ), i.e., $\overline{O S M}$ must be the minimum path length from $O$ to $M$ and, consequently,

$$
\frac{d}{d \theta_{F}} \overline{O S M}=0
$$

where $\theta_{F}$ is the feed-ray angle with respect to the symmetry axis ( $z$-axis), such that $0 \leq \theta_{F} \leq \theta_{E}$. As

$$
\overline{O S M}=r_{F}+\sqrt{\left(x_{M}-x_{S}\right)^{2}+\left(z_{M}-z_{S}\right)^{2}},
$$

where $r_{F}$ is the distance from $O$ to $S, x_{M}$ and $z_{M}$ are the coordinates of $M$, and

$$
\begin{aligned}
& x_{S}=r_{F} \sin \theta_{F} \\
& z_{S}=r_{F} \cos \theta_{F}
\end{aligned}
$$

are the coordinates of $S$, one can show from (1) that

$$
\frac{d r_{F}}{d \theta_{F}}=\frac{r_{F}\left(x_{M} \cos \theta_{F}-z_{M} \sin \theta_{F}\right)}{\overline{O S M}-\left(x_{M} \sin \theta_{F}+z_{M} \cos \theta_{F}\right)},
$$

which is the first-order differential equation to be solved for the reflector surfaces.
In (4), besides $r_{F}$, there are two variables that remain to be determined: the main-reflector coordinates $x_{M}$ and $z_{M}$. That is accomplished by applying the conservation of energy along the ray tube and by enforcing the desired equal path length $\ell_{o}$ from $O$ to the aperture point $A$. However, to render simpler equations it is appropriate to define first an auxiliary Cartesian coordinate system $\left(x^{\prime}\right.$ and $z^{\prime}$, as illustrated in Figs. 1 and 2) to describe the main-reflector coordinates, such that

$$
\begin{aligned}
& x^{\prime}=x \cos \gamma-z \sin \gamma \\
& z^{\prime}=x \sin \gamma+z \cos \gamma .
\end{aligned}
$$

So, with the help of Figs. 1 and 2 one observes that the equal path-length condition is simply enforced by

$$
\ell_{o}=\overline{O S M}-z_{M}^{\prime}+z_{A}^{\prime},
$$

where $z_{M}^{\prime}$ is the $z^{\prime}$-coordinate of the main-reflector point $M$ and $z_{A}^{\prime}$ is the (predetermined) $z^{\prime}$-coordinate of the antenna aperture, which is actually needed just to prevent the aperture from intersecting the antenna symmetry axis and, thus, avoid unnecessary singularities. The coordinate $z_{M}^{\prime}$ is obtained from (6), with the help of (2), (3), and (5).

In turn, the $x^{\prime}$-coordinate of $M$ (i.e., $x_{M}^{\prime}$ ) is determined from the conservation of energy [12]. However, as the ADElike antenna provides the inverse of the feed illumination at the antenna aperture while the ADC-like antenna does not, a distinction must then be made in the shaping algorithm. Note from Fig. 1 that for the ADC-like configuration the conical ray tube emanating from $O$ with semi-angle $\theta_{F}$ is mapped at the aperture with $x^{\prime} \in\left[x_{2}^{\prime}, x_{M}^{\prime}\right]$, while from Fig. 2 one observes that for the ADE-like antenna the mapping occurs with $x^{\prime} \in\left[x_{M}^{\prime}, x_{1}^{\prime}\right]$, where $x_{2}^{\prime}$ and $x_{1}^{\prime}$ are the $x^{\prime}$-coordinates of the main-reflector points $P_{2}$ and $P_{1}$, respectively, given by

$$
\begin{aligned}
& x_{2}^{\prime}=\left(D_{B} / 2\right) \cos \gamma-z_{B} \sin \gamma \\
& x_{1}^{\prime}=x_{2}^{\prime}+W_{A} .
\end{aligned}
$$

So, for the ADC-like antenna, the conservation of energy along the ray tube imposes that

$$
\int_{0}^{\theta_{F}} F(\theta) r_{F}^{2} \sin \theta d \theta=N \int_{x_{2}^{\prime}}^{x_{M}^{\prime}} P\left(x^{\prime}\right) \rho\left(x^{\prime}\right) d x^{\prime},
$$

while for the ADE-like antenna

$$
\int_{0}^{\theta_{F}} F(\theta) r_{F}^{2} \sin \theta d \theta=N \int_{x_{M}^{\prime}}^{x_{1}^{\prime}} P\left(x^{\prime}\right) \rho\left(x^{\prime}\right) d x^{\prime},
$$

where $F(\theta)$ is the circularly-symmetric feed power density, $P\left(x^{\prime}\right)$ is the desired aperture power density,

$$
\rho\left(x^{\prime}\right)=x^{\prime} \cos \gamma+z_{A}^{\prime} \sin \gamma
$$

is the radial distance from the symmetry axis to the antenna aperture, and

$$
N=\left[\int_{0}^{\theta_{E}} F(\theta) r_{F}^{2} \sin \theta d \theta\right] \div\left[\int_{x_{2}^{\prime}}^{x_{1}^{\prime}} P\left(x^{\prime}\right) \rho\left(x^{\prime}\right) d x^{\prime}\right]
$$


is the normalization factor assuring that the feed power captured by the subreflector is conserved at the antenna conical aperture.

Once $x_{M}^{\prime}$ and $z_{M}^{\prime}$ are obtained from (6)-(11), (5) is applied to attain $x_{M}$ and $z_{M}$, which are finally substituted into (4). The subreflector coordinates are then obtained from the numerical solution of (4), with the help of (3) and varying $\theta_{F}$ from 0 to $\theta_{E}$. The first-order non-linear differential equation (4) may be numerically integrated using any widely available method, as the fourth-order RungeKutta method. For that, the initial condition at $\theta_{F}=0$ must be specified. From Figs. 1 and 2 one immediately observes that such condition is

$$
r_{F}\left(\theta_{F}=0\right)=V_{S} .
$$

\section{NumericAl EXAMPLES With A UNIFORM APERTURE ILLUMINATION}

Before proceeding to the shaping and further analysis of omnidirectional dual-reflector antennas designed for maximum gain, we establish classical arrangements that will help in the definition of the initial design parameters. Classical omnidirectional dual-reflector arrangements with $\gamma=90^{\circ}$ are investigated in [8] and [9], where formulas and results for the specification of geometries with relatively large radiation efficiencies are presented. So, from [8] and [9] we chose the classical ADC and ADE arrangements depicted (with dotted lines) in Figs. 3(a) and 4(a), respectively, together with the feed geometry (a coaxial horn). Their relevant characteristics are summarized in Table I. Their omnidirectional radiation patterns are illustrated in Figs. 3(b) and 4(b), respectively. The moment-method (MoM) analyses took into account the complete electromagnetic coupling among reflectors and horn structures. For the aperture-method (ApM) analyses, based on the GO aperture field, the following model was adopted for the electric field radiated from the feed [8],[9]:

$$
\vec{E}_{F}\left(\vec{r}_{F}\right)=\left[\frac{J_{0}\left(k R_{i} \sin \theta_{F}\right)-J_{0}\left(k R_{e} \sin \theta_{F}\right)}{\sin \theta_{F}}\right] \frac{e^{-j k r_{F}}}{r_{F}} \hat{\theta}_{F}
$$

where $J_{0}(x)$ is the zero order Bessel function and $R_{i}=$ $0.45 \lambda$ and $R_{e}=0.9 \lambda$ are the internal and external radii of the coaxial horn aperture, respectively.

Then, the GO shaping formulation of Sect. III was applied for the synthesis of maximum-gain configurations [i.e., with a constant aperture power density $P\left(x^{\prime}\right)$ ] keeping the same values of $W_{A}, \gamma, D_{B}, V_{S}, z_{B}$, and $\theta_{E}$ of their classical counterparts. The feed model of (13) was used to specify the feed power density

$$
F\left(\theta_{F}\right)=\left|\vec{E}_{F}\left(\vec{r}_{F}\right)\right|^{2} /(2 \eta) .
$$

The shaped reflector geometries are depicted (with solid lines) in Figs. 3(a) and 4(a), together with some optical paths from $O$ to the antenna aperture. The radiation patterns are those of Figs. 3(c) and 4(c), from which we observe the gain improvement provided by the reflector shaping. Some of the

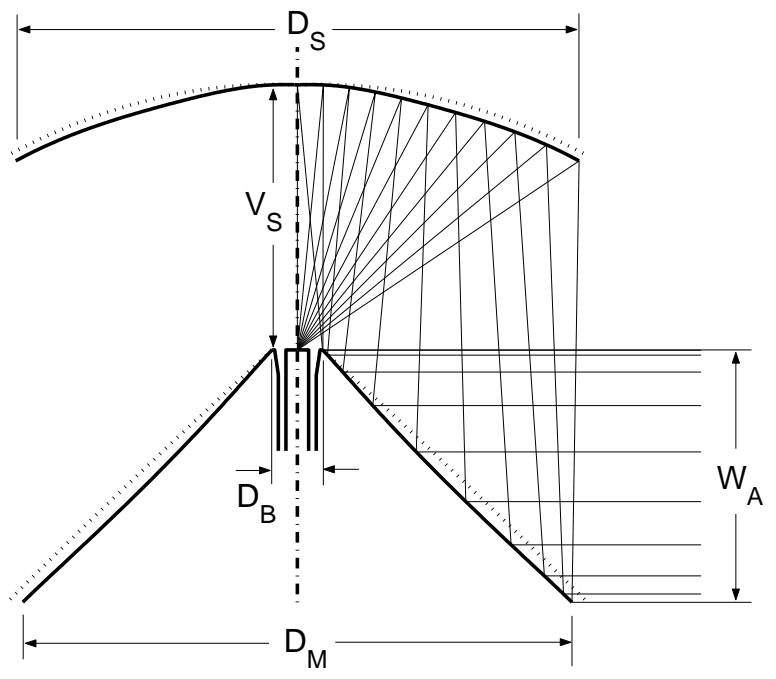

(a)

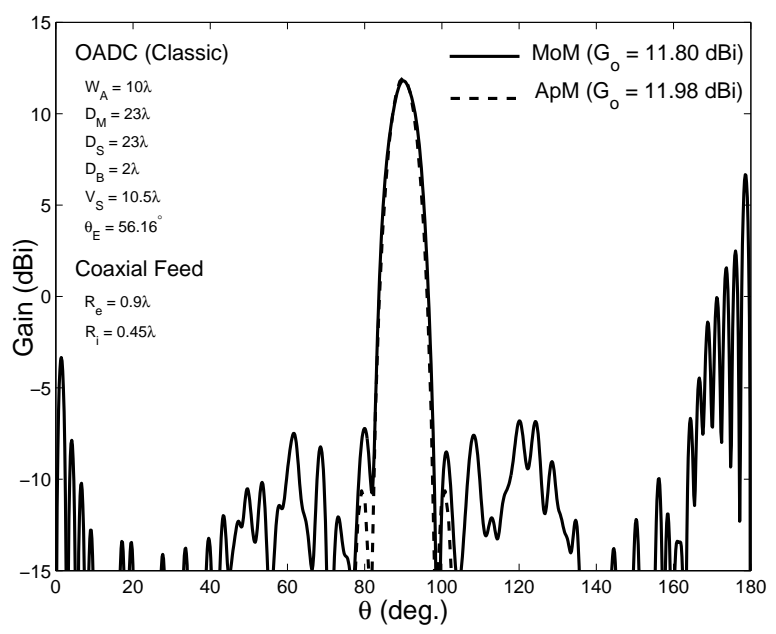

(b)

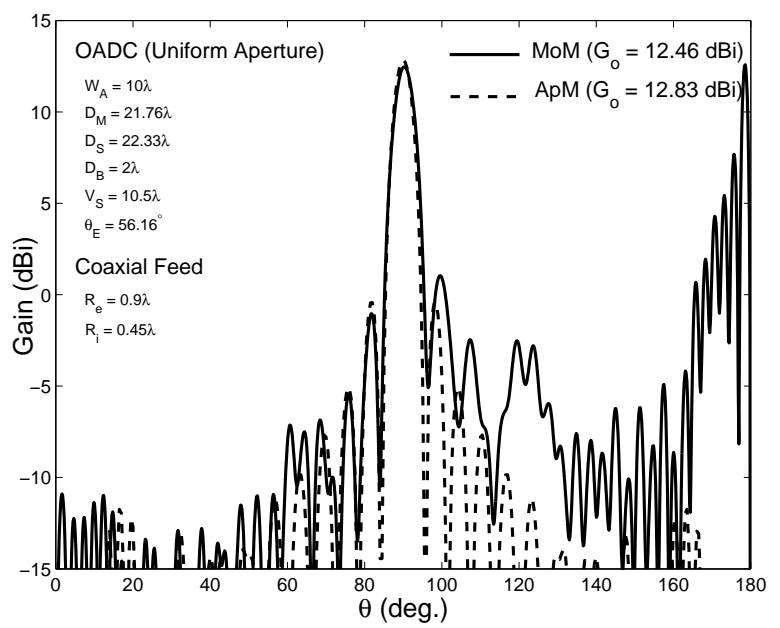

(c)

Fig. 3. Omnidirectional ADC-like antenna shaped for a uniform aperture illumination: (a) classical (dotted lines) and shaped (solid) geometries, and radiation patterns of the (b) classical and (c) shaped geometries.

relevant characteristics of the shaped reflector systems are listed in Table I. 


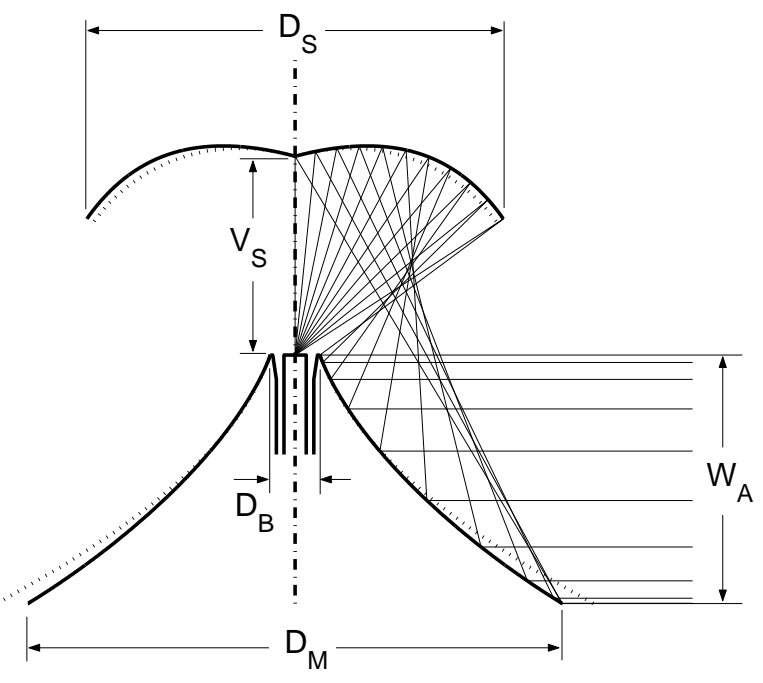

(a)

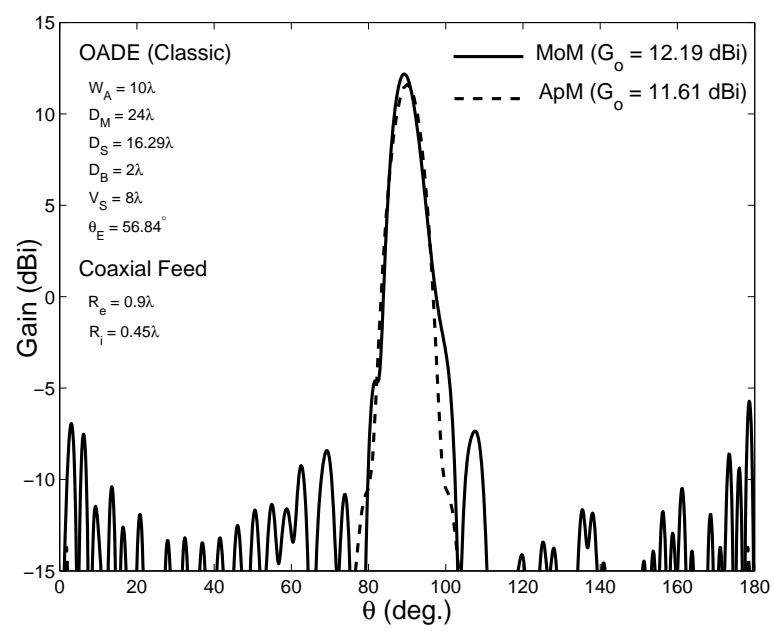

(b)

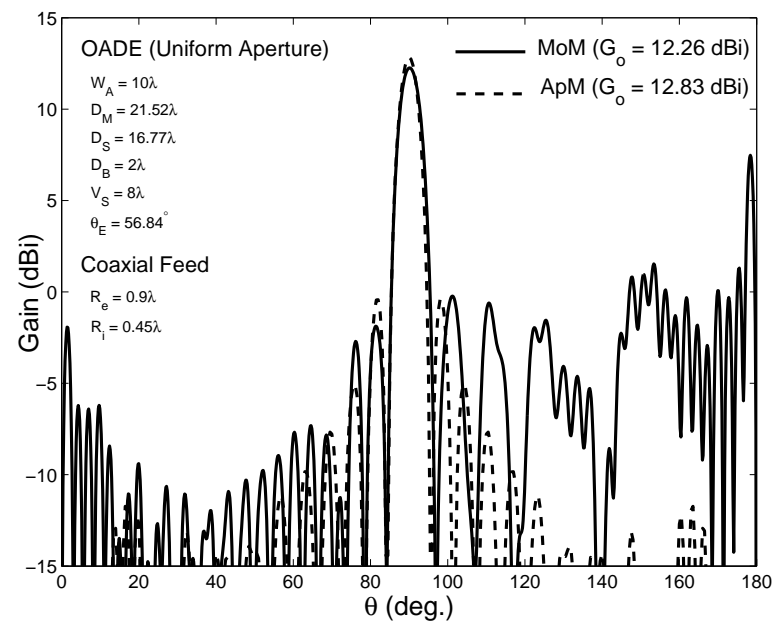

Fig. 4. Omnidirectional ADE-like antenna shaped for a uniform aperture illumination: (a) classical (dotted lines) and shaped (solid) geometries, and radiation patterns of the (b) classical and (c) shaped geometries.

\section{CONClusion}

A GO shaping procedure for maximum gain was developed for dual-reflector antennas, whose body-of-revolution
TABLE I

DUAL-REFlector Antenna CHARACTERISTICS.

\begin{tabular}{c|c|c|c|c}
\hline Characteristic & $\begin{array}{c}\text { Classical } \\
\text { ADC }\end{array}$ & $\begin{array}{c}\text { Shaped } \\
\text { ADC }\end{array}$ & $\begin{array}{c}\text { Classical } \\
\text { ADE }\end{array}$ & $\begin{array}{c}\text { Shaped } \\
\text { ADE }\end{array}$ \\
\hline \hline$W_{A}(\lambda)$ & 10 & 10 & 10 & 10 \\
\hline$\gamma\left({ }^{\circ}\right)$ & 90 & 90 & 90 & 90 \\
\hline$D_{M}(\lambda)$ & 23 & 21.76 & 24 & 21.52 \\
\hline$D_{S}(\lambda)$ & 23 & 22.33 & 16.29 & 16.77 \\
\hline$D_{B}(\lambda)$ & 2 & 2 & 2 & 2 \\
\hline$V_{S}(\lambda)$ & 10.5 & 10.5 & 8 & 8 \\
\hline$z_{B}(\lambda)$ & 0 & 0 & 0 & 0 \\
\hline$\theta_{E}\left({ }^{\circ}\right)$ & 56.16 & 56.16 & 56.84 & 56.84 \\
\hline MoM Gain $(\mathrm{dBi})$ & 11.80 & 12.46 & 12.19 & 12.26 \\
\hline ApM Gain $(\mathrm{dBi})$ & 11.98 & 12.83 & 11.61 & 12.83 \\
\hline
\end{tabular}

surfaces are suited for omnidirectional coverage. The GO shaping was demonstrated for two different reflector configurations, based on the classical ADC and $\mathrm{ADE}$ arrangements. The results were further validated by accurate MoM analyses, accounting for the complete electromagnetic coupling among reflectors and feed structure.

\section{ACKNOWLEDGMENTS}

This work was partially supported by FAPEMIG and CNPq, Brazil.

\section{REFERENCES}

[1] A. P. Norris and W. D. Waddoup, "A millimetric wave omnidirectional antenna with prescribed elevation shaping," in Proc. ICAP - 4th Int. Conf. Antennas and Propagation, pp. 141-145, 1985.

[2] M. Orefice and P. Pirinoli, "Dual reflector antenna with narrow broadside beam for omnidirectional coverage," Electron. Lett., vol. 29, no. 25, pp. 2158-2159, Dec. 9, 1993.

[3] P. Besso, R. Bills, P. Brachat, and R. Vallauri, "A millimetric wave omnidirectional antenna with cosecant squared elevation pattern," in Proc. ICAP - 10th Int. Conf. Antennas and Propagation, vol. 1, pp. 448-451, 1997.

[4] H. B. Abdullah, "A prototype Q-band antenna for mobile communication systems," in Proc. ICAP-10th Int. Conf. Antennas and Propagation, vol. 1, pp. 452-455, 1997.

[5] J. R. Bergmann, F. J. V. Hasselmann, and M. G. C. Branco, ”A singlereflector design for omnidirectional coverage," Microwave Opt. Tech. Lett., vol. 24, no. 6, pp. 426-429, Feb. 14, 2000.

[6] A. G. Pino, A. M. A. Acuña, and J. O. R. Lopez, "An omnidirectional dual-shaped reflector antenna," Microw. Opt. Tech. Lett., vol. 27, no. 5, pp. 371-374, Dec. 5, 2000.

[7] J. R. Bergmann and F. J. S. Moreira, "An omni directional ADE reflector antenna," Microwave Opt. Tech. Lett., vol. 40, no. 3, pp. 250254, Feb. 5, 2004.

[8] F. J. S. Moreira and J. R. Bergmann, "Classical axis-displaced dual-reflector antennas for omnidirectional coverage," accepted for publication in the IEEE Trans. Antennas Propagat.

[9] J. R. Bergmann and F. J. S. Moreira, "Simple design equations for omnidirectional axis-displaced dual-reflector antennas," Microwave Opt. Tech. Lett., vol. 45, no. 2, pp. 159-163, Apr. 20, 2005.

[10] V. Galindo, "Design of dual-reflector antennas with arbitrary phase and amplitude distributions," IEEE Trans. Antennas Propagat., vol. AP-12, no. 4, pp. 403-408, July 1964.

[11] A. Prata, Jr., Lecture Notes of the Graduate Course EE578 - Reflector Antennas, Dept. of Electrical Engineering-Electrophysics, University of Southern California, Spring 1995.

[12] J. J. Lee, L. I. Parad, and R. S. Chu, "A shaped offset-fed dualreflector antenna," IEEE Trans. Antennas Propagat., vol. AP-27, no. 2, pp. 165-171, Mar. 1979 